\title{
Experimental Demonstration of Brokered Orchestration for end-to-end Service Provisioning and Interoperability across Heterogeneous Multi-Operator (Multi-AS) Optical Networks
}

\author{
A. Castro( ${ }^{(1)}$, LI. Gifre ${ }^{(2)}$, C. Chen ${ }^{(3)}$, J. Yin $^{(3)}$, Z. Zhu ${ }^{(3)}$, L. Velasco ${ }^{(2)}$, S. J. B. Yoo ${ }^{(1)}$ \\ (1) University of California (UC Davis), Davis, USA, albcastro@ucdavis.edu \\ (2) Universitat Politècnica de Catalunya (UPC), Barcelona, Spain \\ (3) University of Science and Technology of China (USTC), Hefei, China
}

\begin{abstract}
A$ broker on top of opaquely-managed optical domains advertising their capabilities is proposed to provision multi-AS connections in multi-operator scenarios. In case of no spectrum continuity, intra-domain spectral defragmentation is performed. Experimental assessment was conducted on a distributed multi-continental infrastructure.
\end{abstract}

\section{Introduction}

Flexgrid elastic optical networking (EON) is a promising technique for future metro/core optical networks. To control EONs, Software-defined Networking (SDN) has been widely studied in recent years, in particular when based on the OpenFlow (OF) protocol for its open interface and flexibility in terms of network control and programming. The IETF has been working on a similar approach and recently standardized the Application-Based Network Operations (ABNO) architecture ${ }^{1}$. Previous works on such a software-defined elastic optical networking (SDEON) focused on single/multi-AS scenarios under the single operator premise ${ }^{2}$. However, multi-AS networking architectures are very relevant in real operational scenarios to enhance network scalability and service reach. Therefore, how to support a multi-AS with multiple operators SD-EON is an important topic and needs to be carefully investigated. Note that each operator advertises partial information regarding the topology and connectivity of its AS.

A broker-based SDN solution was proposed in ${ }^{3}$, where a broker is introduced on top of all the SDN controllers to coordinate end-to-end resource management and path provisioning. The centralized broker updates the virtual network topology, manages the resource information of inter-AS links and aggregated (abstracted) intra-AS links, and computes endto-end routing, modulation formats, and spectrum assignment (RMSA) ${ }^{4}$.

Notwithstanding, due to the different dynamicity of each AS, the probability of finding a multi-AS transparent path fulfilling the spectrum continuity constraint might be low. Therefore, per-AS defragmentation can be performed with a global view. In this paper, we propose a mechanism where each AS advertises its internal capabilities, e.g. their ability to implement spectrum defragmentation or any other inoperation planning operation ${ }^{5}$. A planning tool connected to the broker is used to decide the optimal set of operations to provision end-to-end paths.

\section{Broker-based Multi-Operator Architecture}

Let us assume a multi-operator multi-AS flexgrid optical network, where each AS is managed by an SDN/OF controller or an ABNO-based architecture. On top of the ASs, a broker coordinates end-to-end multi-AS provisioning (Fig. 1).

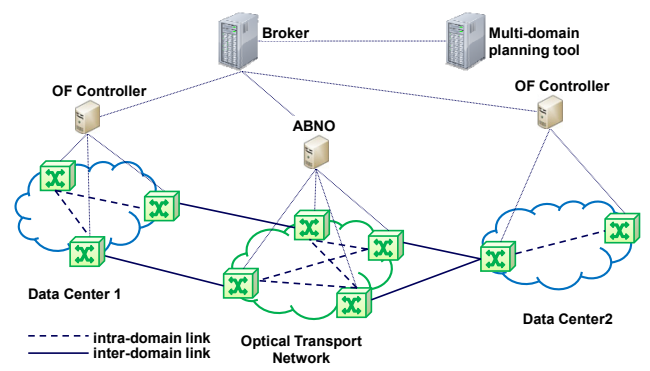

Fig. 1: Multi-AS architecture

Each AS advertises an abstracted intra-AS link information to the broker that depends on both, internal AS policies and the specific agreement with the broker. The broker has a global view of the virtualized network topology, including full information of the inter-AS links and abstracted intra-AS link status gathered from each AS.

In addition, an AS may agree to expose further features to the broker. For example, some ASs may have deployed specific hardware (e.g., wavelength converters/regenerators) and/or implemented optimization algorithms (e.g., spectrum defragmentation algorithms ${ }^{4}$ ), named as capabilities.

To model the underlying data plane, let us assume a graph $\mathrm{G}(N, E)$, where $N$ is the set of optical nodes and $E$ is the set of optical links connecting two nodes. Graph $G$ is structured as a set of ASs $D$. Every AS $d$ consists of three differentiated subset of nodes:

- $N_{e}$ : subset of edge nodes, end-points of demands; 


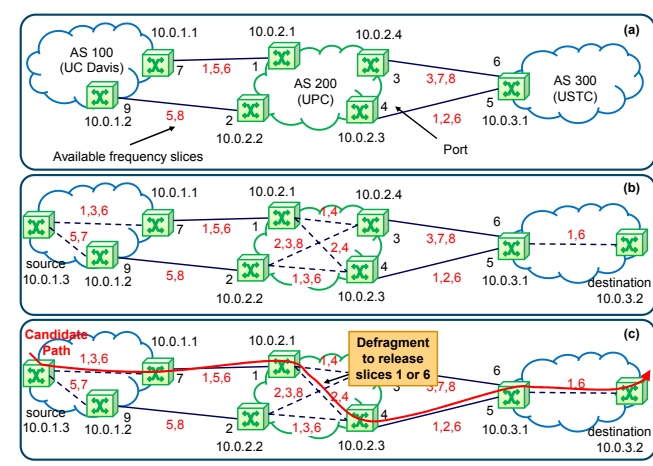

Fig. 2: Example of path computation

- $N_{t}$ : subset of internal AS nodes;

- $N_{i}$ : subset of border AS nodes. Then, $N=N_{e} U$ $N_{t} \cup N_{i}$ with $N_{e} \cap N_{i}=\varnothing$.

- Let $S$ be the set of available frequency slices in each optical link.

Regarding the links, two subsets are considered:

- $E_{i}$ : subset of inter-AS links, connecting two nodes in $N_{i}$ belonging to two different ASs;

- $E_{n}$ : subset of abstracted intra-AS links. Each $e$ $\in E_{n}$ abstracts connectivity between either a node in $N_{e}$ and another node in $N_{i}$ belonging to the request's end $A S s$, or between two nodes in $N_{i}$ belonging to transit ASs.

Each link $e$ is represented by a tuple $<a_{e}, z_{e}, S_{e}$, $c_{e}>$, where $a_{e}, z_{e} \in N_{e} \cup N_{i}$ are the end nodes, $S_{e}$ is the subset of available frequency slices, and $c_{e}$ is the cost.

Since both, broker and the planning tool will be requested to perform complex computations, each AS is assumed to advertise sets $N_{i}$ and $E_{i}$ at start time, and update the set $S$ for each link in $E_{i}$ to follow updates, independently from path computation requests. In addition, each AS advertises its capabilities (e.g., spectrum defragmentation) (Fig. 2a). When a computation is requested, the broker collects intra-AS data $\left(E_{n}\right)$ (Fig. 2b), which are advertised to the planning tool in case that in-operation planning is needed (Fig. 2c).

Fig. 3 illustrates the proposed provisioning workflow, which is divided into three main phases: $i$ ) the Domain Advertisement phase is initiated when the broker first connects to the ASs controllers. The broker collects the inter-AS information, along with the AS's capabilities; ii) the Path Computation phase is triggered by the arrival of a new inter-AS path computation request to an SDN controller. Next, the SDN controller forwards the request to the broker (step 5). Afterwards, the broker gets the intra-AS connectivity (steps 6 and 7). Then, the broker makes a path computation request to the planning tool, adding in the request message the new topology information just obtained (step

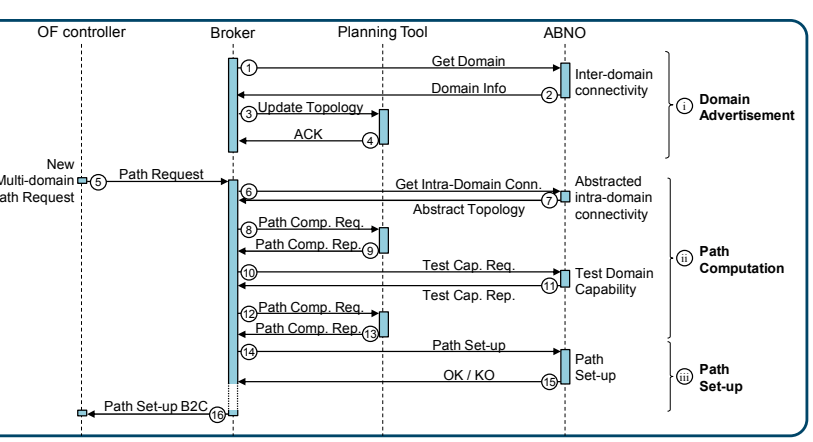

Fig. 3: Proposed workflow

8). If the planning tool finds a feasible solution it responds to the broker the multi-AS path to be set-up. Otherwise, it responds a no-path and proposes a solution using one or more capabilities (step 9). In the latter case, the broker tests if the capabilities are still available (steps 10 and 11). If the capabilities are successfully tested, the broker sends a new path computation request to the planning tool allowing the possibility of the using the just tested capabilities during the computation (step 12). Eventually, the planning tool responds with the multi-AS path to be set-upped and the list of capabilities to be used (step 13); iii) in the Path Set-up phase, the broker, following the solution proposed by the planning tool, instructs the SDN controllers to signal the intra-AS path and configure the borders routers (steps 14 and 15). Once all the SDN controllers finish its local setup, the broker informs the SDN controller which made the original request that the inter-AS path is signaled.

\section{Experimental Assessment}

The experimental validation was carried out on a distributed field trial set-up connecting premises in UC Davis (Davis, California), USTC (Hefei, China), and UPC (Barcelona, Spain) (Fig. 1). The broker, the OF controllers and agents have been developed in Python and run in a computer cluster under Linux. The UPC's Planning tool for optical networks (PLATON) ${ }^{6}$ and the $\mathrm{ABNO}$ has been developed in $\mathrm{C}++$ for Linux.

Regarding the management plane, to enable the broker to orchestrate the experiment, we have developed an HTTP REST API at the broker, which is implemented by the SDN controllers and PLATON. For each API function a specific $\mathrm{XML}$ has been devised. These XML files act as input/output parameters for the API functions (see Fig. 5 and Fig. 6).

Fig. 4 shows the exchanged messages from a broker point of view. For the sake of clarity the numbers of the messages in the figures are in correspondence with each other. 


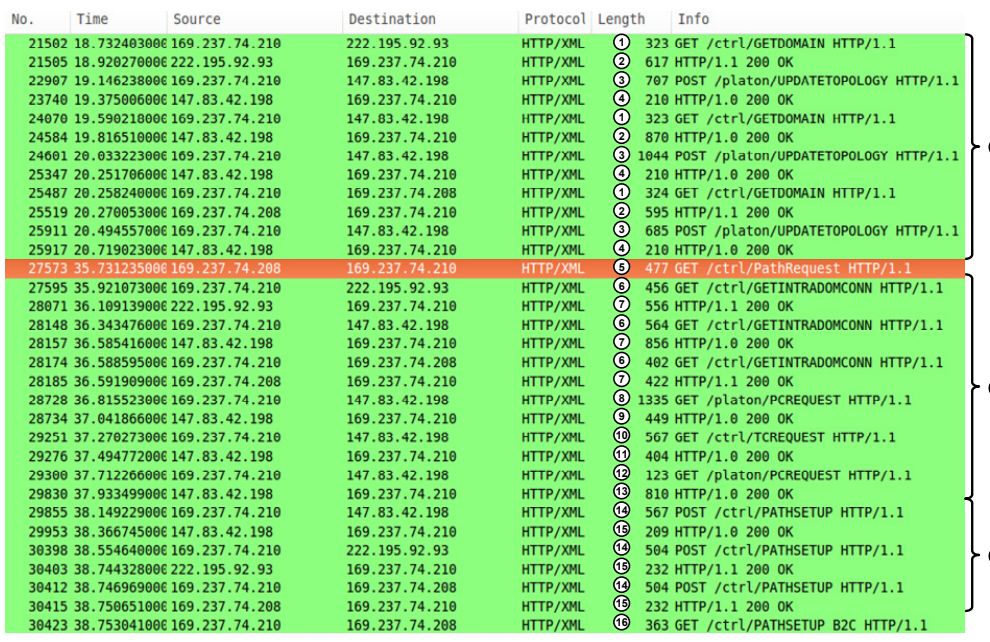

Fig. 4: Messages Exchange at the broker

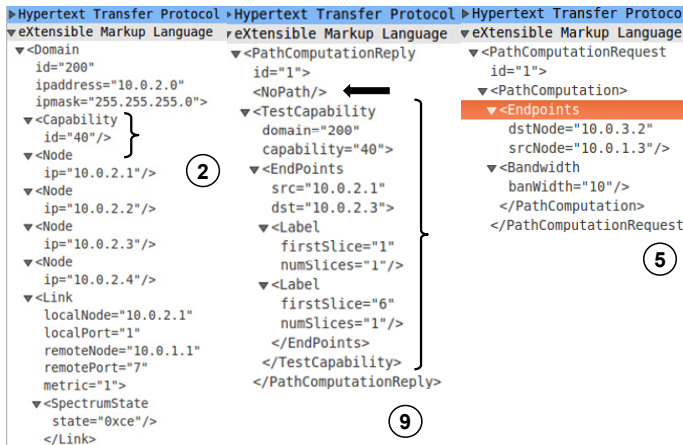

Fig. 6: XML files for steps 2, 5, and 9

The workflow starts when the broker connects to all three SDN controllers and populates its topology. Every time a new topology is obtained, a copy is sent to PLATON, in order to maintain broker and PLATON databases synchronized (steps 1-4). In the event of a path computation request received from a SDN controller (step 5), the Broker collects abstracted intra-AS connectivity and AS capabilities from every controller (steps 6-7). Afterwards, the broker sends a path computation request to PLATON (step 8). In the path computation message, the broker also includes the new topology information just learned. PLATON, first updates its database with the new topology information contained in the request message, and then performs the path computation. Due to our set up, no solution is found. Consequently, a NoPath reply is sent to the broker. Within the reply message PLATON suggests that if defragmentation is used in the UPC AS, a solution can be found (step 9). Then, the broker accepts PLATON suggestion and tests the defragmentation capability in the UPC AS (step 10). As result of the test the UPC AS responds OK (step 11). Immediately after, the broker resends the path computation request to PLATON, but this time informing that the

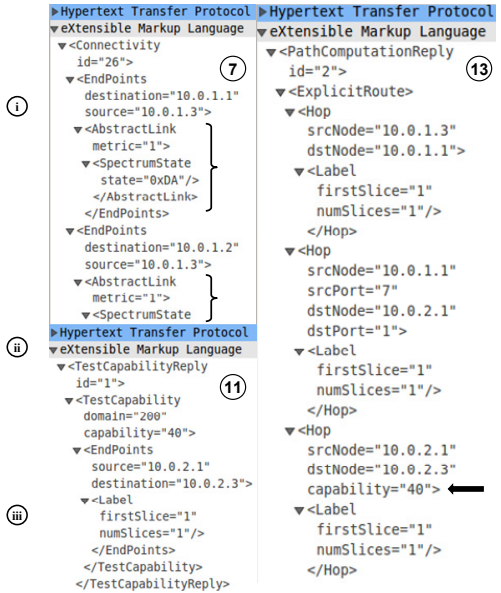

Fig. 5: XML files for steps 7, 11 and 13 defragmentation capability can be used (step 12). Now PLATON finds a solution, and sends it to the broker. The solution in the path computation reply, the XML contains the routing and spectrum allocation, and the capability to be performed (step 13). Finally, the Broker creates the set of configurations to be forwarded to the corresponding SDN controllers (step 14). Eventually, when every controller confirms that the configuration has been set-up (step 15), the broker informs the requester SDN controller that the multi-AS path is signaled (step 16).

\section{Conclusions}

We have experimentally validated a new workflow managed by the broker to provision a multi-AS optical path. Due to the lack of resources, the broker delegates complex inoperation computation to a Planning tool.

Experiments were carried out in a distributed test-bed spanning three continents.

\section{Acknowledgements}

The research leading to these results has received funding from DOE under grant DE-FC02-13ER26154, NSF under EECS grant 1028729, ARL under grant W911NF-14-2-0114, the EC $7^{\text {th }}$ FP under grant 317999 IDEALIST, and from the Spanish MINECO SYNERGY project TEC2014-59995-R.

\section{References}

[1] D. King and A. Farrel, "A PCE-Based Architecture for Application-Based Network Operations," IETF RFC 7491, 2015.

[2] D. King and A. Farrel, "The Application of the Path Computation Element Architecture to the Determination of a Sequence of Domains in MPLS and GMPLS," IETF RFC 6805, 2012.

[3] S. J. B. Yoo, "Multi-domain Cognitive Optical Software Defined Networks with Market-Driven Brokers," Proc ECOC, We.2.6.3, 2014

[4] L. Velasco et al, "Solving Routing and Spectrum Allocation Related Optimization Problems: from OffLine to In-Operation Flexgrid Network Planning," IEEE/OSA JLT, vol. 32, pp. 2780-2795, 2014.

[5] L. Velasco, et al., "In-Operation Network Planning," IEEE ComMag, vol. 52, pp. 52-60, 2014.

[6] LI. Gifre et al, "Experimental Assessment of a High Performance Back-end PCE for Flexgrid Optical Network Re-optimization," Proc. OFC, W4A.3, 2014 